\title{
Electron Tunneling from Graphene
}

\author{
V.A.Fedirko \\ Moscow State University of Technology "Stankin” (MGTU "Stankin”), Moscow, Russia \\ National Research University of Electronic Technology "MIET” (MIET), Moscow, Russia \\ *Corresponding Author: vfed@mail.com
}

Copyright (C) 2014 Horizon Research Publishing All rights reserved.

\begin{abstract}
The paper reports on theoretical estimation of quantum electron tunneling rate from graphene sheet into some 3D media through a rectangular potential barrier. Tunneling from a lateral sheet surface and from its edge has been considered. We use perturbation theory and Bardeen transfer Hamiltonian approach to cope with the problem. The results show that tunneling current density from a free edge of a graphene may exceed greatly that from its lateral surface. That can explain observed higher emission current from carbon nanotubes with open edge compared to closed edge nanotubes.
\end{abstract}

Keywords Graphene, Quantum tunneling, Graphene Edge

\section{Introduction}

Since the publication of K. S. Novoselov et al. [1] graphene "has rapidly changed its status from being an unexpected and sometimes unwelcome newcomer to a rising star" [2] and has firmly established itself in condensed matter physics. It is a unique material considered as perspective one for various applications (see, e.g. [2]).

Lately graphene has attracted interest as potential candidate for effective electron field emitters with rather low turn-on voltage and high emission current [3-5]. For further development theoretical study of electron field emission from graphene is meaningful as the conventional Fowler-Nordheim (FN) theory is not directly applicable in that case and does not describe the experimental data quite adequately. Quantum electron tunneling is a key phenomenon in the process of field emission. However the of electron tunneling out from graphene into vacuum (or into a normal metal) is not fairly understood yet. An attempt has been undertaken in [6], but the authors' approach based on Klein tunneling model [7, 8] does not seem quite irreproachable as it does not take into account the fact that the electron wave functions in vacuum outside graphene layer obey free-electron-like 3D Hamiltonian rather than the Dirac-like Hamiltonian [1], so chiral tunneling [8] can't take place in that case. The results of $[9,10]$ based on density-functional theory calculations, though rather interesting, make problem in matching with conventional theory for electronic properties of graphene [11]. In this paper we suggest a model for electron tunneling out from graphene into 3D media in the framework of standard formalism for envelope wave functions of electronic excitations in 2D graphene details of which can be found elsewhere (see, e.g. the review article [11]).

\section{Tunneling Through a Lateral Surface of a Graphene Sheet}

Here we consider the quantum electron tunneling from graphene monolayer to $3 \mathrm{D}$ environment through a rectangular potential barrier at the lateral surface of an infinite graphene sheet. The electron in graphene are described by $2 \mathrm{D}$ two-component wave functions

$$
\begin{aligned}
& {\left[\begin{array}{l}
\varphi_{A}(x, y) \\
\varphi_{B}(x, y)
\end{array}\right]=\frac{1}{\sqrt{2}}\left[\begin{array}{l}
1 \\
\eta
\end{array}\right] \cdot e^{i k_{x} x+i k_{y} y},} \\
& \eta=\frac{k_{x}+i k_{y}}{k}=e^{i \theta}, \quad 0 \leq \theta \leq \pi
\end{aligned}
$$

assuming the sheet is at $x 0 y$ coordinate plane which are the envelope amplitude on sublattices $A$ and B of honeycomb 2D graphene lattice and are eigenfunctions of the 2D Weyl-Dirac- like Hamiltonian $[1,11]$ :

$$
\hat{H}=-i \hbar v_{0} \sigma \cdot \nabla
$$

where $v_{0} \approx 10^{8} \mathrm{~cm} / \mathrm{c}$ is the characteristic velocity (usually called Fermi velocity), $\sigma=\left(\sigma_{x}, \sigma_{y}\right)$ are the Pauli matrices.

To solve the above tunneling problem one needs to know the wave functions behavior outside graphene sheet. To estimate it we approximate a graphene layer with a $\delta$-like quantum potential well $-\Lambda \delta(z)$. We thus be able to extend the $2 \mathrm{D}$ graphene wave functions in $z$-direction simply by corresponding boundary conditions at $z= \pm 0$ on outer side of graphene sheet surfaces [12]. We define the "strength" of the potential $\Lambda$ using the envelope wave function inside a solitary free-standing graphene sheet for matching conditions. Assuming wave functions in free space outside graphene (both for sublattice $A$ and $B$ of graphene sheet) as 


$$
\psi_{A, B}(\boldsymbol{r}) \sim \varphi_{A, B}(x, y) \cdot e^{-\kappa_{0}|z|}
$$

one thus easily finds:

$$
\Lambda=\frac{\hbar^{2} \kappa_{0}}{m}
$$

Here

$$
\kappa_{0}^{2}=-2 m \varepsilon_{K} / \hbar^{2}-2 m v_{0} k_{\|} / \hbar+k_{\|}^{2}, \quad \boldsymbol{k}_{\|}=\left(k_{x}, k_{y}\right)
$$

$\varepsilon_{K}<0$ being the electron energy near a Dirac $\boldsymbol{K}$-point of 2D Brillouin zone of graphene [11], measured from zero vacuum level, $m$ is he electron mass, $v_{0}-$ the above characteristic velocity for the linear electron energy spectrum in graphene:

$$
\varepsilon_{k_{\|}}=\hbar v_{0} k_{\|}
$$

Typically

$$
\frac{\hbar k_{\|}}{2 m v_{0}}<<1
$$

so we can put:

$$
\kappa_{0}^{2} \approx-2 m \varepsilon_{K} / \hbar^{2}-2 m v_{0} k_{\|} / \hbar
$$

It is worth noting that due to the peculiarities of electron energy spectra in graphene, the "strength" $\Lambda$ of the $\delta$-potential has proved $k_{\|}$-dependent

Let now the potential barrier on the both sides of the sheet has finite width $l$ as shown schematically in fig. 1 .

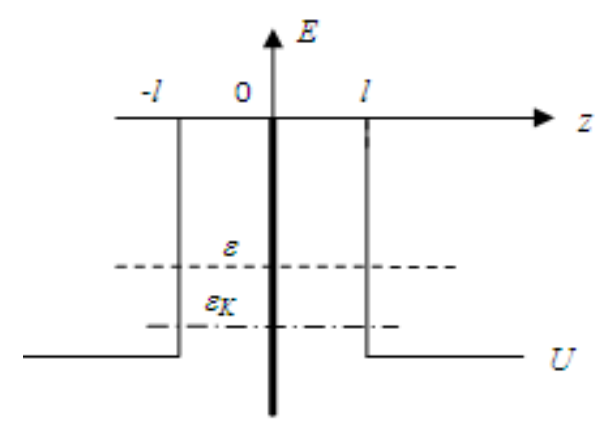

Figure 1. Simplified energy diagram of a potential barrier

We seek for the wave functions of an electron outside graphene sheet in the form:

$$
\psi_{A, B}(\boldsymbol{r})=\left\{\begin{array}{l}
\varphi_{A, B}(x, y) \cdot\left[A e^{-\kappa z}+B e^{\kappa z}\right], 0<z<l \\
C \varphi_{A, B}(x, y) \cdot e^{i k_{z}(z-L)}, z>l \\
\psi(-z)=\psi(z)
\end{array}\right.
$$

(both for sublattices $A$ and $B$ as well), where $k_{\mathrm{z}}$ is found from the equation:

$$
U+\frac{\hbar^{2} k_{\|}^{2}}{2 m}+\frac{\hbar^{2} k_{z}^{2}}{2 m}=\varepsilon_{K}+\hbar v_{0} k_{\|}
$$

$U$ being the potential energy right from the barrier .
Matching (4) at $z=0$ and $z=l$ :

$$
\begin{aligned}
& \psi(+0)=\psi(-0)=\psi(0) \\
& \psi^{\prime}(+0)-\psi^{\prime}(-0)=-2 \kappa_{0} \psi(0) \\
& \psi(l+0)=\psi(l-0) \\
& \psi^{\prime}(l+0)=\psi^{\prime}(l-0)
\end{aligned}
$$

gives together with (4):

$$
\begin{aligned}
& \left(\kappa-\kappa_{0}\right) A-\left(\kappa+\kappa_{0}\right) B=0 \\
& A e^{-\kappa l}+B e^{\kappa l}-C=0 \\
& -\kappa A e^{-\kappa l}+\kappa B e^{\kappa l}-i k C=0
\end{aligned}
$$

and results in the equation for $\kappa$ :

$$
\left(\kappa-i k_{z}\right)\left(\kappa-\kappa_{0}\right) e^{\kappa l}=\left(\kappa+\kappa_{0}\right)\left(\kappa+i k_{z}\right) e^{-\kappa l}
$$

For weak tunneling, $\kappa l>>1$, one has approximately:

$$
\kappa^{2} \approx \kappa_{0}^{2}+\left(4 \frac{\kappa_{0}^{2}-k_{z}^{2}}{\kappa_{0}^{2}+k_{z}^{2}}+8 i \frac{\kappa_{0} k_{z}}{\kappa_{0}^{2}+k_{z}^{2}}\right) \cdot \kappa_{0}^{2} \cdot e^{-2 \kappa_{0} l}
$$

and:

$$
C / A \approx \frac{2 \kappa_{0}}{\kappa_{0}-i k_{z}} e^{-\kappa_{0} l}
$$

To find the partial tunneling rate per a $\boldsymbol{k}_{\|}$-electron state in graphene

$$
\nu_{s}\left(\boldsymbol{k}_{\|}\right)=2 \frac{\hbar k_{z}}{m}|C|^{2}
$$

we can put $|\mathrm{A}|^{2} \approx \kappa_{0}$ and thereby obtain from (8):

$$
\nu_{s}\left(\boldsymbol{k}_{\|}\right)=\frac{8 \hbar \kappa_{0}^{3} k_{z}}{\left(\kappa_{0}^{2}+k_{z}^{2}\right) m} e^{-2 \kappa_{0} l}
$$

For small $k_{z}<<\kappa_{0}$ eq.(9) may be reduced to

$$
\nu_{s}\left(\boldsymbol{k}_{\|}\right) \approx \frac{8 \hbar \kappa k_{z}}{m} e^{-2 \kappa_{0} l}, \quad k_{z}<<\kappa_{0}
$$

while for $k_{z} \sim \kappa_{0}$ it gives

$$
\nu_{s}\left(\boldsymbol{k}_{\|}\right) \approx \frac{8 \hbar \kappa_{0}^{2}}{2 m} e^{-2 \kappa_{0} l}, k_{z} \sim \kappa_{0}
$$

The total tunneling current is found by summing over all $\boldsymbol{k}_{\|}$states near two equivalent $\boldsymbol{K}$-points in graphene with corresponding occupation numbers $n\left(\boldsymbol{k}_{\|}\right)$:

$$
J=\sum_{\boldsymbol{k}_{\|}} n\left(\boldsymbol{k}_{\|}\right) \cdot \nu_{s}\left(\boldsymbol{k}_{\|}\right) \Rightarrow \frac{S}{(2 \pi)^{2}} \cdot g \int n\left(\boldsymbol{k}_{\|}\right) \nu_{s}\left(\boldsymbol{k}_{\|}\right) d \boldsymbol{k}_{\|}
$$

where $S$ is the area of a tunneling contact, $g=4$ is the degeneracy factor.

It is worthwhile to draw one's attention to the fact that the energy states in graphene are quasi-stationary now. If, similarly to (3), identify $\hbar^{2} \kappa^{2} / 2 m$ with the energy $\varepsilon$ of such a quasi-stationary state

$$
\frac{\hbar^{2} \kappa^{2}}{2 m} \approx-\varepsilon
$$


it appears complex, $\operatorname{Im} \varepsilon / \hbar$ being the decay factor. We then can find $\nu\left(\boldsymbol{k}_{\|}\right)$from the continuity equation

$$
\frac{d}{d t} \int_{-\infty}^{+\infty}\left|\Psi_{\boldsymbol{k}_{\|}}\right|^{2} d z+\nu_{S}\left(\boldsymbol{k}_{\|}\right)=0,|\Psi|^{2} \approx \kappa_{0} \cdot e^{-2 \kappa_{0} z+\frac{2 \operatorname{Im} \varepsilon}{\hbar} t}
$$

which again leads to (8).

In case the tunneling takes place from one of the sheet surface only, the tunneling current will be a half of its value (9). Though that is rather evident it can be found by direct solution of the corresponding boundary problem as well.

\section{Tunneling Through an Edge of a Graphene Sheet}

In this section we consider electron tunneling from the edge of a graphene sheet through a rectangular potential barrier. In this paper we confine our study to a zigzag edge of a honeycomb graphene lattice (see fig. 2).

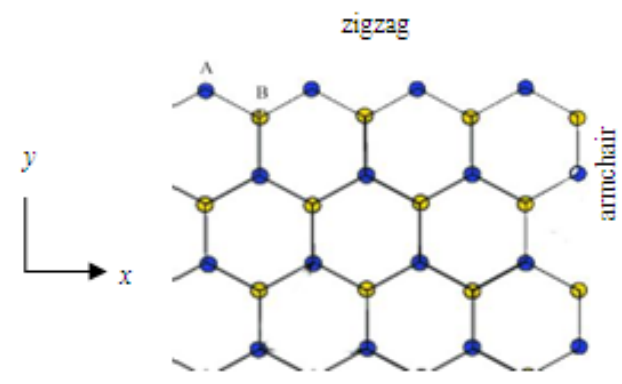

Figure 2. A fragment of a graphene sheet lattice with both zigzag and armchair edges displayed

A grapheme sheet is supposed macroscopically long at $x$-direction and at $y<0$. The zero barrier potential (fig. 1) is now endless on the both sides of the sheet unless $y<l$, and falls to $U<0$ at $y>l$ as shown schematically in fig. 3. We consider the case of weak tunneling so we assume that $l>>d$, where $d$ is the graphene layer thickness.

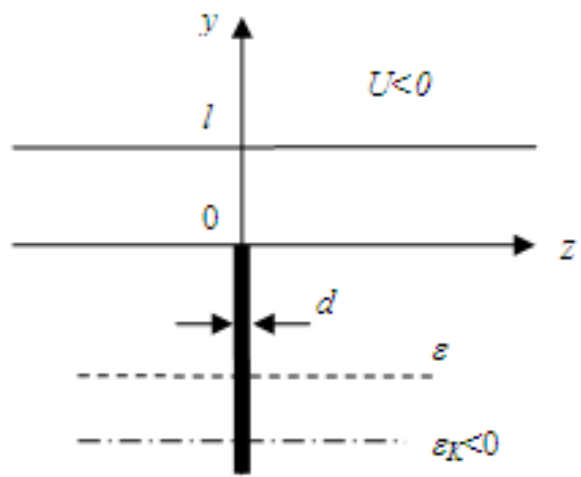

Figure 3. Schematic view of graphene edge

We use the Bardeen transfer Hamiltonian approach [13] to cope with that tunneling problem. To estimate the tunneling matrix element

$$
M=\frac{\hbar^{2}}{2 m} \int_{S} d s\left(\Psi_{G} \frac{\partial \Psi^{*}}{\partial n}-\frac{\partial \Psi_{G}}{\partial n} \Psi^{*}\right)
$$

we perform the integration over the outside surface $S$ of the sheet, so $\Psi_{G}$ and $\Psi$ are "undisturbed" electron wave functions for graphene and for external environment respectively in the barrier region just at the outer side of the sheet surface.

For the external wave function we use the well-known expression for an electron plane wave reflecting from endless rectangular barrier (see, e.g. [14]):

$$
\Psi(x, z) \approx \frac{e^{i k_{x} x+i k_{z} z}}{\sqrt{L_{x} L_{y} L_{z}}} \cdot\left\{\begin{array}{l}
t e^{\kappa(y-l)}, 0<y<l \frac{2 q}{q+i \kappa} \\
e^{i q(y-l)}+r^{\prime} e^{-i q(y-l)}, l<y<L_{x} \\
t=\frac{2 q}{q+i \kappa}, \quad r^{\prime}=\frac{q-i \kappa}{q+i \kappa}
\end{array}\right.
$$

where $L_{x}, L_{y}, L_{z}$ - are the external environment dimensions which are supposed macroscopic, and $\kappa$ is the sub-barrier decay factor:

$$
\kappa=\sqrt{\left.-2 m E / \hbar^{2}+k_{z}^{2}+k_{y}^{2}\right)}, E \geq U
$$

$E<0$ being the electron energy.

Further on, we shall be interested in the tunneling from the edge only, so we restrict integration in (10) over the sheet thickness $|z|<d / 2$ in $y=+0$ plane. We intend to use the envelope electron wave function in graphene to estimate $M$ value. Matching conditions must ensure the continuity of the electron current at the edge. (Note in brackets that this guarantees hermicity of the Hamiltonian). As a zigzag edge does not mix electron states from different $\boldsymbol{K}$-valleys [11] the above requirement is satisfied if one puts, taking into account that a zigzag edge is formed by $A$-sublattice (Fig. 2):

$$
\begin{aligned}
& \left.\Psi_{G}(x, y=+0, z)\right|_{|z|<d / 2}=\phi_{A}(x, y=-0) / \sqrt{d} ; \\
& \left.\frac{\partial \Psi_{G}}{\partial y}(x, y=+0, z)\right|_{|z|<d / 2}=\frac{2 m v_{0}}{i \hbar \sqrt{d}} \phi_{B}(x, y=-0)
\end{aligned}
$$

where $\phi_{A}$ and $\phi_{B}$ are the envelope wave functions for sublattices $A$ and $B$ respectively in free-standing graphene half-plane. With (13) the electron current through the edge per unit length along $x$-direction at $y=+0$ is:

$$
\begin{aligned}
& \left.j\right|_{y=+0}=\left.d \cdot \frac{i \hbar}{2 m}\left(\Psi_{G} \frac{\partial \Psi_{G}^{*}}{\partial y}-\Psi_{G}^{*} \frac{\partial \Psi_{G}}{\partial y}\right)\right|_{\substack{y=+0 \\
z \mid<d / 2}}= \\
& =\left.v_{0}\left(\phi_{A} \cdot \phi_{B}^{*}+\phi_{A}^{*} \cdot \phi_{B}\right)\right|_{y=-0} \\
& =\left.v_{0}\left(\Psi_{G} \sigma_{y} \Psi_{G}^{*}\right)\right|_{y=-0}=\left.j\right|_{y=-0}
\end{aligned}
$$

which coincides with the linear current density at inner side in graphene $j(y=-0)$ :

$$
\left.j\right|_{y=-0}=\left.v_{0}\left(\psi \sigma_{y} \psi\right)\right|_{y=-0}
$$

$\psi$ being the two component $(A, B)$ amplitude [15]. They are: 


$$
\begin{aligned}
& \phi_{A}(x, y)=\frac{1}{\sqrt{2}}\left(L_{G x} L_{G y}\right)^{-1 / 2}\left(e^{i k_{y} y}+r e^{-i k_{y} y}\right) e^{i k_{x} x} \\
& \phi_{B}(x, y)=\frac{1}{\sqrt{2}}\left(L_{G x} L_{G y}\right)^{-1 / 2}\left(\eta e^{i k_{y} y}+\eta^{*} r e^{-i k_{y} y}\right) e^{i k_{x} x} \\
& \eta=\frac{k_{x}+i k_{y}}{k}=e^{i \theta}, \quad 0 \leq \theta \leq \pi
\end{aligned}
$$

where $L_{G x}$ and $L_{G y}$ are the grapheme sheet macroscopic dimensions (further on we put $L_{G x}=L_{x}$ ).

The tunneling rate for a given $\boldsymbol{k}_{\|}$-state of an electron in graphene is given by the well-known perturbation theory golden rule:

$$
\nu_{e}=\frac{2 \pi}{\hbar} \sum_{q, k_{z}}\left|M\left(k_{x}, k_{y}\right)\right|^{2} \delta\left(E\left(q, k_{y}, k_{z}\right)-E_{G}\left(k_{x}, k_{y}\right)\right)
$$

where $E_{\mathrm{G}}\left(k_{x}, k_{y}\right)$ is the electron energy in grapheme for given $\left(k_{x}, k_{y}\right)$ state (see eq. 3 ) and $E\left(q, k_{y}, k_{z}\right)$ is the energy of a transmitted electron outside the barrier:

$$
\begin{aligned}
& E_{G}\left(k_{x}, k_{y}\right)=\varepsilon_{K}+\hbar v_{0}\left(k_{x}^{2}+k_{y}^{2}\right), \\
& E\left(q, k_{y}, k_{z}\right)=U+\frac{\hbar^{2}}{2 m}\left(q^{2}+k_{y}^{2}+k_{z}^{2}\right) ; \\
& \varepsilon_{K}<0, U<0 .
\end{aligned}
$$

With (10) - (14) in mind one has for $|M|^{2}$ :

$$
\mid M\left(\left.\left(\boldsymbol{k}_{\|}\right)\right|^{2}=\left.\frac{4 d q^{2} e^{-2 \kappa l}}{L_{z}\left(q^{2}+\kappa^{2}\right)} \cdot\left(\frac{\hbar^{2}}{2 m}\right)^{2}\left(\kappa^{2}\left|\phi_{A}\right|^{2}+\left(\frac{2 m v_{0}}{\hbar}\right)^{2}\left|\phi_{B}\right|^{2}+\frac{2 m v_{0} \kappa}{\hbar} \cdot \operatorname{Re}\left(\phi_{A} \phi_{B}^{*}\right)\right)\right|_{y=0}\right.
$$

In (17) we have taken into account that due to the factor $e^{-2 \kappa l}$ only small values of $k_{z} \sim(\kappa / l)^{1 / 2}<<1 / d$ are actual in (15) as follows from (12) with $\kappa d \sim 1$ and $d<<l$. Now note that $\operatorname{Re}\left(\phi_{A} \phi^{*}{ }_{B}\right)=0$ as the electron current through the edge $j_{y}$ $\sim \operatorname{Re}\left(\phi_{A} \phi^{*}{ }_{B}\right)$ is absent in zero approximation. Also $|r|=1$ in zero approximation, so we can put $r=e^{i \alpha}$. With that we find at $y=0$ :

$$
\left|\varphi_{A}\right|^{2} \sim \cos ^{2}(\alpha / 2),\left|\varphi_{B}\right|^{2} \sim \cos ^{2}(\theta-\alpha / 2)
$$

For high enough barrier $\alpha \sim \pi$, and we have from (18), that in zero approximation at $y=-0$ :

$$
\left|\varphi_{A}\right|^{2} \approx 0,\left|\varphi_{B}\right|^{2} \sim \sin ^{2} \theta
$$

in accordance with [11].

So, taking into account that also $2 m c^{2}>\hbar^{2} \kappa^{2} / 2 m$ we can neglect the first term in eq. (15) too, witch gives:

$$
|M|^{2} \approx \frac{8 \hbar^{2} q^{2} v_{0}^{2} d e^{-2 \kappa l} \sin ^{2} \theta}{L_{y} L_{G y} L_{z}\left(q^{2}+\kappa^{2}\right)}
$$

Converting the sum in (14) to the integration with usual procedure and performing the integration over $q$ with $\delta$-function we come to the expression:

$$
\nu_{e}\left(\boldsymbol{k}_{\|}\right) \approx \frac{32 m v_{0}^{2} d \sin ^{2} \theta}{\pi \hbar q_{0}^{2} L_{G y}} \cdot \int_{0}^{k_{0}} d k_{z} \cdot \sqrt{q_{0}^{2}-k_{z}^{2}} \cdot e^{-2 \kappa l},
$$

where

$$
k_{0}^{2}=2 m / \hbar^{2}\left[\varepsilon_{K}-U+\hbar v_{0}\left(k_{x}^{2}+k_{y}^{2}\right)-\frac{\hbar^{2} k_{x}^{2}}{2 m}\right]
$$

(see also eqs. 2, 3). For small $q_{0}<<\kappa_{0}$ (21) the integration results in

$$
\nu_{e}\left(\boldsymbol{k}_{\|}\right) \approx \frac{8 m v_{0}^{2} d \sin ^{2} \theta}{\hbar L_{G y}} e^{-2 \kappa_{0} l}, \quad q_{0}<<\kappa_{0}
$$

and for $q_{0} \sim \kappa_{0}$ in

$$
\nu_{e}\left(\boldsymbol{k}_{\|}\right) \approx \frac{32 m v_{0}^{2} d \sin ^{2} \theta}{\hbar \sqrt{\pi \kappa_{0} l} L_{G y}} e^{-2 \kappa_{0} l}, \quad q_{0} \sim \kappa_{0}
$$

The total tunneling current is found like in section 2 by summing over all $\boldsymbol{k}_{\|}$states with corresponding occupation numbers $n\left(\boldsymbol{k}_{\|}\right)$:

$$
J=\sum_{\boldsymbol{k}_{\|}} n\left(\boldsymbol{k}_{\|}\right) \cdot \nu_{e}\left(\boldsymbol{k}_{\|}\right) \Rightarrow \frac{L_{G y} L_{G x}}{(2 \pi)^{2}} g \int n\left(\boldsymbol{k}_{\|}\right) \nu_{e}\left(\boldsymbol{k}_{\|}\right) d \boldsymbol{k}_{\|}
$$

It is interesting to compare the partial current density resulting from the expressions (19) and (2). In case $k_{z} \sim k_{0}<<$ $\kappa_{0}$, one finds

$$
\frac{\nu_{e}\left(\boldsymbol{k}_{\|}\right)}{\nu_{s}\left(\boldsymbol{k}_{\|}\right)} \sim \frac{m v_{0}^{2}}{\varepsilon_{K}} \cdot \frac{\kappa_{0}}{k_{z}}>>1,
$$

as $\left(m v_{0}^{2} / \varepsilon_{\mathrm{K}}\right) \sim 2$ for graphene. When $\mathrm{k}_{\mathrm{z}} \sim \mathrm{k}_{0} \sim \kappa_{0}$,

$$
\frac{\nu_{e}\left(\boldsymbol{k}_{\|}\right)}{\nu_{s}\left(\boldsymbol{k}_{\|}\right)} \sim \frac{4 m v_{0}^{2}}{\varepsilon_{K} \sqrt{\pi \kappa_{0} l}}
$$

though $\kappa_{0} l>1$, even in that case $j_{e}\left(\boldsymbol{k}_{\|}\right) / j_{s}\left(\boldsymbol{k}_{\|}\right)>1$ if $\left(\kappa_{0} l\right)$ is not too large: $\kappa_{0} l<10$. It may be the reason for higher emission current from open edge carbon nanotube than from closed edge nanotube, reported elsewhere [16].

\section{Summary}

We have studied quantum electron tunneling from graphene sheet into some 3D media through a rectangular potential barrier. Based on perturbation theory and Bardeen transfer Hamiltonian approach we have made theoretical estimation for tunneling rate both from a lateral sheet surface and from its edge. The results show that tunneling current density from a free edge of a graphene sheet may exceed greatly that from a lateral surface. That can explain observed higher emission current from carbon nanotubes with open edge than from closed edge nanotubes.

\section{Acknowledgements}

The work is supported by the Russian Foundation for Basic Research, Project No. 12-01-00339-a and Project No. 14-01-00663-a. 


\section{REFERENCES}

[1] K. S. Novoselov, A. R. Geim., S. V. Morozov, D. Jiang, Y. Zhang, S. V. Dubonos, I. V. Grigorieva, A. A. Firsov. Electric Field Effect in Atomically Thin Carbon Films, Science, Vol. 306, 666-969, 2004.

[2] A. K. Geim. Graphene: Status and Prospects, Science 324, 1530-1534, 2009.

[3] Goki Eda, H. Emrah Unalan, Nalin Rupesinghe, et al. Field emission from graphene based composite thin films, Appl. Phys. Lett. Vol. 93, 233502 (2008).

[4] Zhong-Shuai Wu, Songfeng Pei, Wencai Ren, et al. Field Emission of Single-Layer Graphene Films Prepared by Electrophoretic Deposition, Adv. Mater., Vol. 21, 1756-1760, 2009.

[5] U. A. Palnitkar, R. V. Kashid, M. A. More, et al. Remarkably low turn-on field emission in undoped, nitrogen-doped, and boron-doped graphene, Appl. Phys. Lett. Vol. 97, 063102, 2010.

[6] S. Sun, L. K. Ang, D. Shiffler, and J. W. Luginsland. Klein tunnelling model of low energy electron field emission from single-layer graphene sheet, Appl. Phys. Lett., Vol. 99, 013112, 2011.

[7] O. Klein. Zeitschrift für Physik, Die Reflexion von Elektronen an einem Potentialsprung nach der relativistischen

Dynamik von Dirac, v. 53, No. 3-4, 157-165, 1929

[8] M. I. Katsnelson, K. S. Novoselov, and A. R. Geim. Chiral tunnelling and the Klein paradox in graphene, Natur. Phys, v. 2, No. 9, 620-625, 2006.

[9] K. Tada and K. Watanabe. Ab Initio Study of Field Emission from Graphitic Ribbons, Phys. Rev. Lett. Vol. 88, 127601, 2002.

[10] Masaaki Araidai, Yasuhiro Nakamura, and Kazuyuki Watanabe. Field emission mechanisms of graphitic nanostructures, Phys. Rev. B, Vol. 70, 245410, 2004.

[11] A. H. Castro Neto, F. Guinea, N. M. R. Peres, K. S. Novoselov, and A. R. Geim. The electronic properties of grapheme, Rev. Mod. Phys., vol. 81, 109-162, 2009.

[12] S. Flugge. Practical Quantum Mechanics I, Springer-Verlag, Berlin - Heidelberg - New-York, 1971.

[13] Bardeen, J. Tunneling from a many-particle point of view, PRL, vol. 6, No.2, pp.57-59, 1961.

[14] L.D.Landau, E.M. Lifshitz. Quantum Mechanics: Non-Relativistic Theory (Volume 3), 3-d edition, Elsevier Butterworth-Heinemann, 1977.

[15] C. W. J. Beenakker. Colloquium: Andreev reflection and Klein tunneling in graphene, Rev. Mod. Phys., v. 80, No.4, 1337-1354, 2008 (arXiv:0710.3848v2 [cond-mat.mes-hall]).

[16] A.V. Eletski. Carbon nanotubes and their emission properties. Physics - Uspekhi, vol. 45, N 4, 369 - 402, 2002. 